\title{
Characterization of VGA liquid crystal panels with partial phase-only modulation: application to pattern recognition
}

S. Vallmitjana, Ignasi Juan Labastida, Estela MartinBadosa, Arturo Carnicer, Ignacio Juvells

S. Vallmitjana, Ignasi Juan I. Labastida, Estela Martin-Badosa, Arturo Carnicer, Ignacio Prades Juvells, "Characterization of VGA liquid crystal panels with partial phase-only modulation: application to pattern recognition," Proc. SPIE 10296, 1999 Euro-American Workshop Optoelectronic Information Processing: A Critical Review, 1029605 (2 June 1999); doi:

10.1117/12.365915

EDent: Euro-American Workshop on Optoelectronic Information Processing, 1999, Colmar, France 


\title{
Characterization of VGA liquid crystal panels with partial phase-only modulation. Application to pattern recognition.
}

\author{
S. Vallmitjana*, I. Labastida, E. Martín-Badosa, A. Carnicer, I. Juvells \\ Universitat de Barcelona, Departament de Física Aplicada i Òptica, \\ Av. Diagonal 647, E08028 Barcelona, Spain
}

\begin{abstract}
The inclusion of optoelectronic displays has enabled optical correlators to improve radically and maintain the competitive edge of optical processors among information processing fields. In this work, an optical correlator has been implemented by using two VGA liquid crystal displays as spatial light modulators, both at the input and Fourier planes. These devices have been removed from a commercial videoprojector and have been characterized in order to operate in different configurations. This characterization is based on an interferometric procedure which includes amplitude and phase modulation measurements. For a phase-only modulation we have compared the use of linearly and elliptically polarized light. In this latter case we have found an operating curve with phase-only modulation which takes values from 0 to $\pi$ radians. This is a limitation when a pure phase filter is required as in data security and encryption, in wavelet-based systems, and in optical pattern recognition, among others. An application in this latter field is presented in our work as a solution to overcome this limited phase depth. We propose a modification of the classical phase-only filter based on the use of the absolute value of the phase. Experimental correlation results using the optical setup and the modified filter have been obtained.
\end{abstract}

Keywords: Pattern recognition, optical correlation, spatial light modulators, liquid crystal displays, phase modulation.

\section{INTRODUCTION}

Nowadays, the presence of liquid crystal displays (LCD) as spatial light modulators (SLM) [1] has become the usual way to display images and control the setup in optical information processing systems [2-4]. Although high frame.rate modulators are available in the market today, a possible way to obtain LCD panels is by removing them from relatively low cost commercial videoprojectors $[5,6]$. These devices are designed for imaging purposes so they present high resolution although operate at video frame rate. Despite several correlators having been designed to work at high frame rate [7], there are some applications that require higher resolution. For instance, adaptive systems based on the joint transform correlation are less critical in terms of video rate but require more pixels to display both scenes. High resolution modulators are also required in broad-area search problems [8].

* Email: santi@optica.ub.es; Tel: +34 934021202; Fax: +34 934021142

1999 Euro-American Workshop Optoelectronic Information Processing: A Critical Review, edited by Philippe Refregier, Bahram Javidi, Proc. of SPIE Vol. 10296 (Vol. CR74), 1029605 (c) (1999) 2017 SPIE · CCC code: 0277-786X/17/\$18 · doi: 10.1117/12.365915 
72 / Critical Reviews Vol. CR74

Generally, amplitude and phase LCD modulation are coupled, in the sense that both amplitude and phase of light on its way through the panel are modified [9]. A relevant configuration with amplitude and phase modulation is the high contrast mode which is often used. However, it is possible to find some operation conditions in which one of them remains mainly constant as the other one changes considerably. These are called amplitude-mostly configuration and phase-mostly configuration [10]. Using a phase-mostly configuration with high LCD constant transmittance maximizes the light efficiency of the optical arrangement, because light absorption is minimum. This operating mode is needed to display phase-only filters as for instance in data security and encryption [11]. It is then necessary to calibrate the device performance, both as a transmittance or amplitude modulator or as a phase modulator $[12,13]$. An easy phase calibration method, based on the fringe analysis of a Mach-Zehnder interferometer, is presented. An amplitude characterization that profits part of the same setup is also explained. Other procedures that extract the phase measurements from optical diffraction information have been developed $[14,15]$.

Two VGA LCDs removed from a commercially available Epson videoprojector (EMP-3000) have been characterized in order to be used in an optical correlator for pattern recognition purposes. The optical setup is based on a converging VanderLugt correlator with one LCD at the input plane working in a high contrast mode and another one in a phase-mostly configuration at the Fourier plane.

By using linearly polarized light we have obtained a good high contrast operating curve but we have not been able to find a suitable phase-mostly configuration. Nevertheless, with elliptically polarized light an only phase modulation mode has been achieved, although the phase just reaches $\pi$ radians.

In order to display a phase-only filter on partial phase modulation devices, we demonstrate that successful correlations can be obtained by taking the absolute value of the phase.

The paper is organized as follows: in Section 2 we describe the optical correlator. In Section 3 we describe a method for characterizing liquid cristal displays and its application to study the response of the two panels used. In Section 4 we suggest a way to overcome the phase limitations of these devices, whereas in Section 5, optical results are carried out. Finally, the conclusions can be found in Section 6 .

\section{OPTOELECTRONIC SETUP}

The converging VanderLugt correlator is a modification of the classical $4 \mathrm{f}$ architecture [16]. The main problem that presents the $4 \mathrm{f}$ correlator is the need of large distances to work in the optical bench with the suitable elements and the appropriate scale. The optical set up of the converging correlator is sketched in Figure 1, [17].

This correlator is based on the coupling of two diffractometers with converging lenses. With this architecture the required distances are shorter than for the $4 \mathrm{f}$ system. It is still possible to further reduce the distances using diverging lenses [1820]. This architecture has another advantage in that it allows the modification of the scale of the Fourier transforms without changing neither the lenses nor the distances between them. This versatility simplifies the fitting between the optical transform 


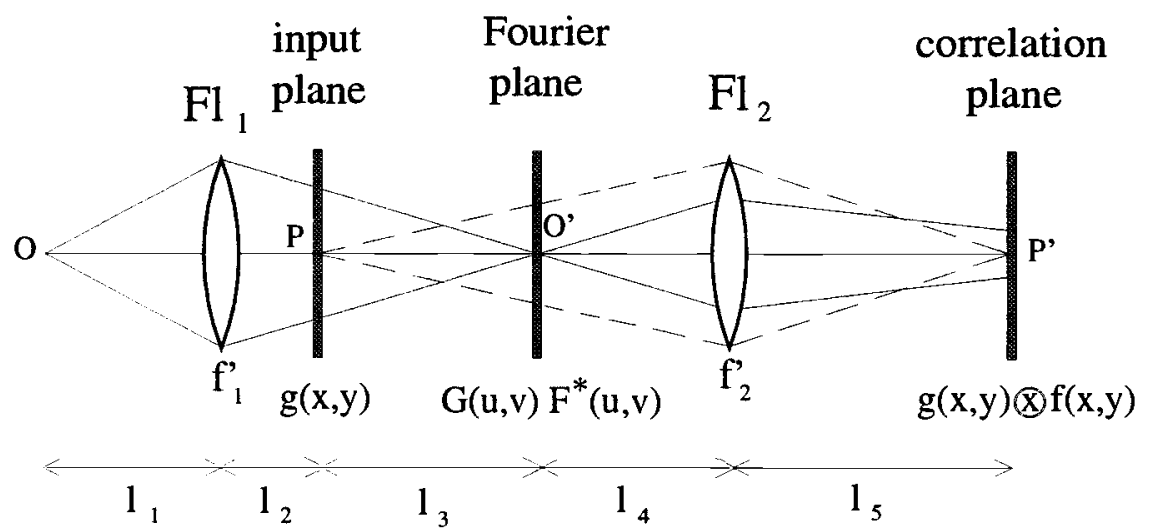

Figure 1. Converging VanderLugt correlator

and the filter. The matching has to be done accurately because the correlation results are extremely sensitive to misaligments [21]. Our optical correlator has been implemented with telephoto systems reducing the overall distance from the expander to the correlation plane to about one meter.

According to Figure 1 it is possible to describe how the converging correlator works. The system is illuminated with a monochromatic light of wavelength $\lambda$ expanded from point source $O$. The scene, denoted by the two-dimensional function $g(x, y)$, is placed after the first converging lens at an arbitrary distance. The Fourier transform of the scene, $G(u, v)$, multiplied by a quadratic phase, appears centered at the point $\mathrm{O}^{\prime}$, image point of $\mathrm{O}$ through the first lens. In this position, or Fourier plane, it is where the filter, $F^{*}(u, v)$, is placed and where the product of the two functions, $G(u, v) F^{*}(u, v)$, is optically obtained. The second lens forms the image of the scene at a plane passing through $\mathrm{P}^{\prime}$, image point of point $\mathrm{P}$. The Fourier transform of the product appears at this plane, thus producing the correlation between the two functions $g(x, y)$ and $f(x, y)$. This plane is called the correlation plane.

In order to study the scales involved in the correlator it is possible to do a mathematical analysis of the system [20,22]. In a first order approximation, a spherical lens with a focal length $f^{\prime}$ can be thought of as a a system which introduces a phase factor $\psi\left(x, y ;-F^{\prime}\right)=e^{-i \frac{\pi}{\lambda f}\left(x^{2}+y^{2}\right)}$. If the scene is denoted by $g(x, y)$, the amplitude distribution at the Fourier plane is:

$$
\mathcal{A}\left(x^{\prime}, y^{\prime}\right)=Q \int \psi\left(-\alpha,-\beta ; L_{1}\right) \psi^{*}\left(\alpha, \beta ; F_{1}^{\prime}\right) \psi\left(\alpha-x, \beta-y ; L_{2}\right) g(x, y) \psi\left(x-x^{\prime}, y-y^{\prime} ; L_{3}\right) d \alpha d \beta d x d y
$$

where $Q$ is a constant and $(\alpha, \beta)$ are the coordinates of the plane where the first lens is placed. Integrating with respect to $\alpha$ and $\beta$ and, after a little of algebra, 
equation 1 reduces to:

$$
\mathcal{A}\left(x^{\prime}, y^{\prime}\right)=Q \psi\left(x^{\prime}, y^{\prime} ; L_{3}\right) \int \psi\left(x, y ; L_{2}+L_{3}-\frac{L_{2}^{2}}{L_{1}-F_{1}^{\prime}+L_{2}}\right) g(x, y) e^{-i \frac{2 \pi L_{3}}{\lambda}\left(x x^{\prime}+y y^{\prime}\right)} d x d y
$$

If $\psi\left(x, y ; L_{2}+L_{3}-\frac{L_{2}^{2}}{L_{1}-F_{1}^{\prime}+L_{2}}\right)=1$, then $\mathcal{A}\left(\boldsymbol{x}^{\prime}, y^{\prime}\right)$ will be the Fourier transform of $g(x, y)$, except for the unimportant constant factor $Q$. This condition is fullfilled when the plane $\left(x^{\prime}, y^{\prime}\right)$ is placed at the position $O^{\prime}$, image point of $O$ :

$$
\psi\left(x, y ; L_{2}+L_{3}-\frac{L_{2}^{2}}{L_{1}-F_{1}^{+}+L_{2}}\right)=1 \quad \rightarrow \quad \frac{1}{f_{1}^{\prime}}=\frac{1}{l_{1}}+\frac{1}{l_{2}+l_{3}}
$$

then:

$$
\mathcal{A}\left(x^{\prime}, y^{\prime}\right)=Q \psi\left(x^{\prime}, y^{\prime}, L_{3}\right) G(u, v) \quad \text { where } \quad u=\frac{L_{3} x^{\prime}}{\lambda} \quad \text { and } \quad v=\frac{L_{3} y^{\prime}}{\lambda}
$$

In an analogous way, we derive the amplitude distribution at the correlation plane $\left(\mathrm{x}^{\prime \prime}, \mathrm{y} "\right)$ :

$$
\begin{array}{r}
\mathcal{C}\left(x^{\prime \prime}, y^{\prime \prime}\right)=L_{4} L_{5} \int \mathcal{A}\left(x^{\prime}, y^{\prime}\right) F^{*}\left(x^{\prime}, y^{\prime}\right) \psi\left(x^{\prime}-\alpha^{\prime}, y^{\prime}-\beta^{\prime} ; L_{4}\right) \psi^{*}\left(\alpha^{\prime}, \beta^{\prime} ; F_{2}^{\prime}\right) \\
\psi\left(\alpha^{\prime}-x^{\prime \prime}, \beta^{\prime}-y^{\prime \prime} ; L_{5}\right) d \alpha^{\prime} d \beta^{\prime} d x^{\prime} d y^{\prime}
\end{array}
$$

where $F^{*}\left(x^{\prime}, y^{\prime}\right)$ represents the filter. In order to reduce the latter expression to a Fourier transform the following condition has to be met:

$$
\frac{1}{f_{2}^{\prime}}=\frac{1}{l_{5}}+\frac{1}{l_{3}+l_{4}}
$$

i.e. the correlation plane is found at the image plane of the scene through the second lens, $F_{2}$. Finally, the amplitude distribution is:

$$
\begin{array}{r}
\mathcal{C}\left(x^{\prime \prime}, y^{\prime \prime}\right)=Q \psi\left(x^{\prime \prime}, y^{\prime \prime} ; L_{5}-\frac{L_{5}^{2}}{L_{4}+L_{5}-F_{2}^{\prime}}\right) \int G\left(L_{3} x^{\prime}, L_{3} y^{\prime}\right) F^{*}\left(x^{\prime}, y^{\prime}\right) \\
e^{-i \frac{2 \pi}{\lambda} \frac{-L_{4} L_{5}}{L_{4}+L_{5}-F_{2}^{\prime}}\left(x^{\prime} x^{\prime \prime}+y^{\prime} y^{\prime \prime}\right)} d x^{\prime} d y^{\prime}
\end{array}
$$

which is, aside of non-detectable phase factors, the Fourier transform of the product of the two functions. This transform produces the correlation between the scene, $g(x, y)$, and the image reference, $f(x, y)$.

The LCDs used in this work have been removed from an Epson EMP-3000 videoprojector. Each videoprojector has three LCDs, one for each RGB channel. In the correlator we use only two of them, one at the input plane and the other one at the Fourier plane. These devices have $640 \times 480$ pixels and their pixel pitch is $42 \times 42 \mu \mathrm{m}$. Figure 2 presents an scheme of the LCD pixel structure.

The own videoprojector electronics addresses the two LCDs by means of a VGA card that stores a 24 -bit image of $640 \times 480$ pixels. This image can be split in three different 8-bit images, one for each RGB channel. We have chosen the blue and red 


\section{Epson VGA-LCD Pixel structure}
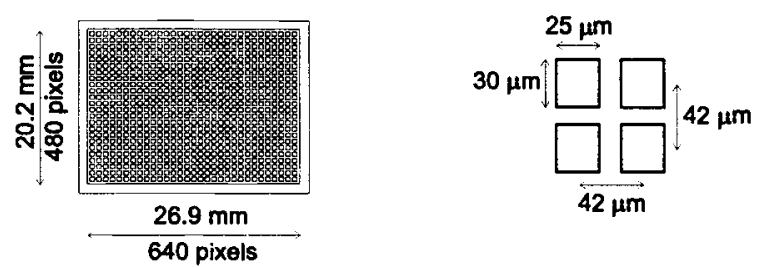

Figure 2. Schematic drawing of the LCD structure.

panels because they present an equivalent design, consequence of the geometry in the original equipment. The green panel is functionaly different, so it is rejected. Therefore, the information corresponding to the scene is sent through the blue channel while the red one is used for the filter. Despite of the two images have 256 grey levels, the videoprojector electronics only takes into account the first five bits of each byte. The reduction to 32 grey levels is not significant in terms of correlation performance as reported [23].

\section{CHARACTERIZATION OF LIQUID CRYSTAL DISPLAYS}

The characterization of the LCDs mainly consists of the obtention of their operating curves, which give information on the way in which these devices modulate light depending on the grey level assigned to each pixel $[13,15,24,25]$. The light can be modulated in amplitude, in phase or in both sinultaneously [10]. This complex transmittance depends on the polarization state of light and on the addressing voltage applied to each pixel. When placing an LCD between two polarizers (polarizer and analyzer) different configurations of the device, that give rise to the different operating curves, depend on the position of the polarizers as well as on the potentiometer control positions (bright, contrast and color) of the videoprojector. When a single videoprojector is used to address two LCDs the aforementioned controls have to be set in a compromise position and the final operating curve adjustement has to be carried out by rotating the polarizers. The whole characterization process includes both the amplitude and the phase modulation measurements.

\subsection{Experimental setup}

The experimental setup used to measure the phase modulation of the LCD is based on a Mach-Zehnder interferometer, as shown in Figure 3. The collimated He-Ne laser beam is splitted by the first beamsplitter (BS1). A CCD camera registers the interference fringes of the two plane-waves when the beam joins again. The phase variation produced when a LCD is placed in one arm of the interferometer is translated onto a fringe displacement on the interference plane [26]. The $\lambda / 2$ plate in the other arm compensates the light polarization changes as it traverses the panel, so maximum contrast interference fringes are obtained. Both the videoprojector and the camera are connected to a computer, where it is possible to change the input SLM image and to analyze the output fringe pattern. The possible grey level values in the computer are comprised between 0 and 255. The LCD modulation depends 
76 / Critical Reviews Vol. CR74

on the orientation of the two polarizers, the position of the potentiometer controls available on the videoprojector and, basically, on the PC input image grey level (gl). When all these variables but the last one remain constant, it is interesting to study the phase and amplitude modulation of the light traversing the device as the input grey level changes, in order to determine the modulation of the LCD when a grey-level image is displayed on it. The method consists on the measurement of the relative phase shift between several pairs of grey levels displayed on the panel: half of the screen always remains with a constant grey level value, $g \mathrm{l}=0$, which acts as a reference (phase modulation zero), and different values are displayed on the other half.

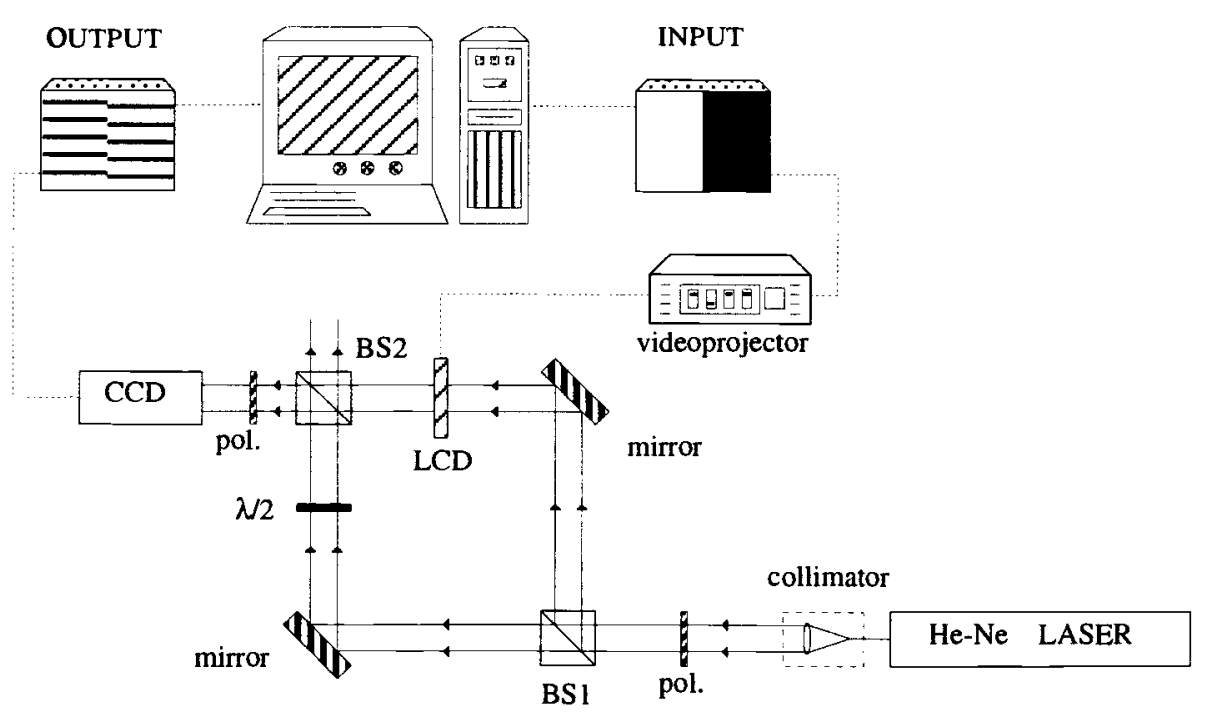

Figure 3. Mach-Zehnder interferometer for phase measurement

\subsection{Phase measurement method}

A typical fringe pattern is shown in Figure 4, where we can see two cosinusoidal interference fringes out of phase, the left one (l) corresponding to the reference level $(\mathrm{gl}=0)$ and the right one $(\mathrm{r})$ to a grey level of 64 . The pixels of the LCD are also visible. The whole digitized picture has 512 rows and columns, as indicated. Because the interference fringes are aligned in the $\mathrm{x}$ or horizontal direction, it is possible to represent their vertical profiles by the 1-dimensional mathematical expressions that follow:

$$
\left\{\begin{array}{l}
I_{l}(y)=a_{l}(y)+b_{l}(y) \cos \left[2 \pi y / P+\phi_{l}(y)\right] \\
I_{r}(y)=a_{r}(y)+b_{r}(y) \cos \left[2 \pi y / P+\phi_{r}(y)\right]
\end{array} \quad(y=0 \ldots N-1)\right.
$$

$y$ is a discrete variable taking $N$ different values, where $N$ is the number of rows $(N=512) . P$ is the period of the fringes, which is related to their frequency by $f=N / P$, while $a_{i}(y)(i=l, r)$ stand for possible background nonuniformities and $b_{i}(y)$ are related to the local contrast of the patterns. Equations 8 work for each 
column and should be approximately equal for all of them. In order to reduce the nonuniformities and with the final idea of having an average measurement, we have determined the mean value of the digitized intensity for each row, considering the central 128 columns (comprised between the two vertical white lines depicted in Figure 4) for both the left and the right fringes. The corresponding distributions are shown in Figure $4 \mathrm{l}$ and $4 \mathrm{r}$. The phase shift to determine is given by:

$$
\phi=\phi_{r}(y)-\phi_{l}(y)=2 \pi \Delta / P
$$

where $\Delta$ represents the right-left fringe displacement. One possibility is to apply Fourier Transform fringe-pattern analysis methods [27], so $\phi_{r}(y)$ and $\phi_{l}(y)$ can be determined separately and then the phase shift can be obtained by performing the subtraction, as indicated in equation 9. Due to the specific characteristics of the present work measurements, it is only necessary to know the phase difference. Consequently, it is easier to calculate the relative fringe displacement and the fringe period and compute $\phi=2 \pi \Delta / P$.
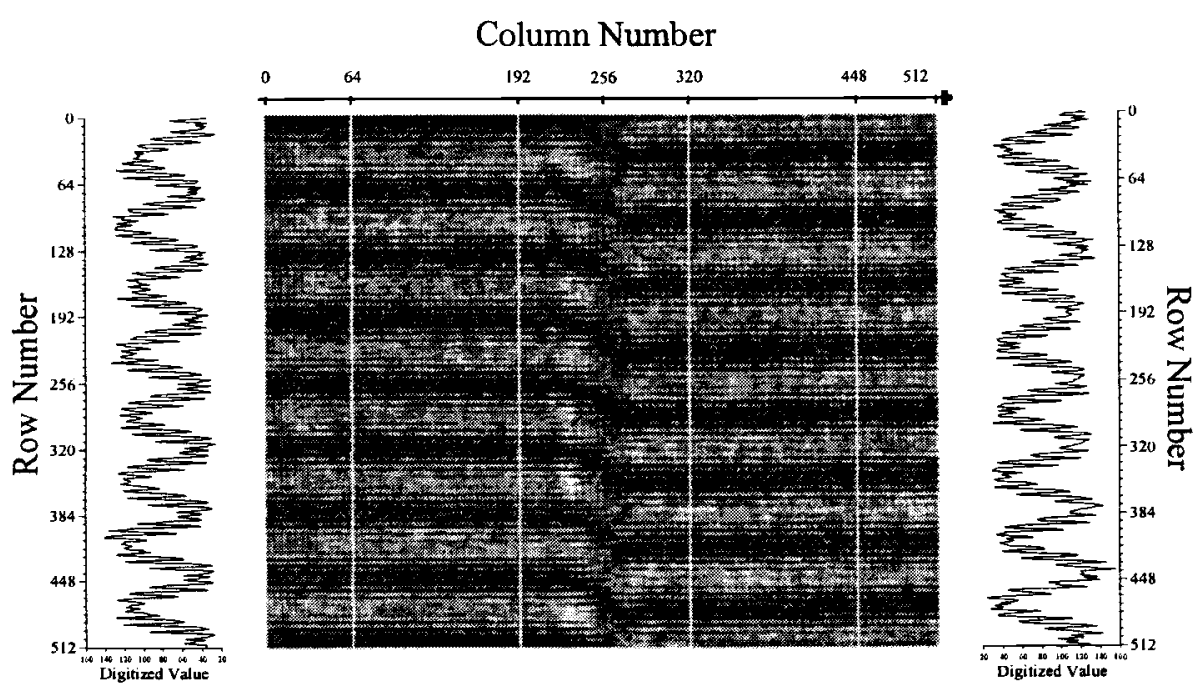

(l)

(r)

Figure 4. Fringe pattern

\subsubsection{Fringe displacement measurement}

A simple method to determine the fringe displacement is to perform the onedimensional correlation product between the two fringe patterns, which can be expressed as:

$$
C_{r l}\left(y^{\prime}\right) \equiv I_{r} \otimes I_{l}=\sum_{k=0}^{k=N-1} I_{r}(k) I_{l}\left(k-y^{\prime}\right)
$$


It gives a measure of the similarity of the two functions as one of them is displaced over the other. The position of the correlation maximum corresponds to the maximum overlapping conditions and, in the case of fringe correlation, it directly provides the fringe displacement.

The correlation product of Equation 10, normalized to its maximum value (100), is depicted in Figure 5. The exact determination of the position of the maximum is difficult, because of the noisy ripple present in the signal, which is primarily due to the LCD pixel structure visible in the fringe patterns (Figure 4).

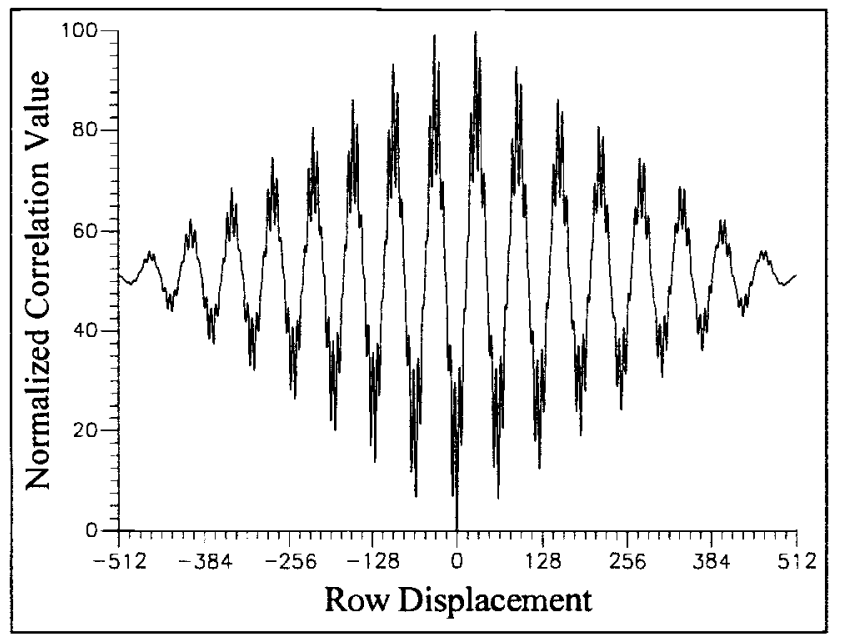

Figure 5. Correlation between the signals in Figures $4 \mathrm{l}$ and $4 \mathrm{r}$

In order to obtain a smoother function, the intensity interference distributions of Figure 4(l) and 4(r) are approximated to a cosines function. We use a Fourier transform technique in which a low-pass filtering is applied to the Fourier transform of one of the signals and, by doing an inverse Fourier transformation, a recovered smoother signal is obtained. Figure 6 shows the amplitude of the Fourier transform of the function in Figure 4(l). The thicker line is the result of applying a low-pass filtering with a cut-off frequency a little above the maximum. The two secondary frequencies, of about 66 and 83 (period of 8 and 6, respectively) correspond to the LCD pixels, to be filtered. The recovered smoother signal for the left fringe distribution is plotted in Figure 7.

The correlation product of the left and right smoothed signals is shown in Figure 8. The maximum location process is easier and gives more accurate results. The correlation function of two infinite sequences having the same period is periodic itself, so a series of equally spaced maxima should appear. When the input signals are finite, as the one plotted in Figure 7, their correlation product has a tendency to decrease as it approaches the edges of the functions, because less non-zero points contribute to the summation of Equation 10. This introduces an error in the maximum position determination. So the graph in Figure 8 has several maxima with a decreasing amplitude as they are faraway from the origin, besides they are not exactly separated one period. In order to reduce measurement errors, an average 


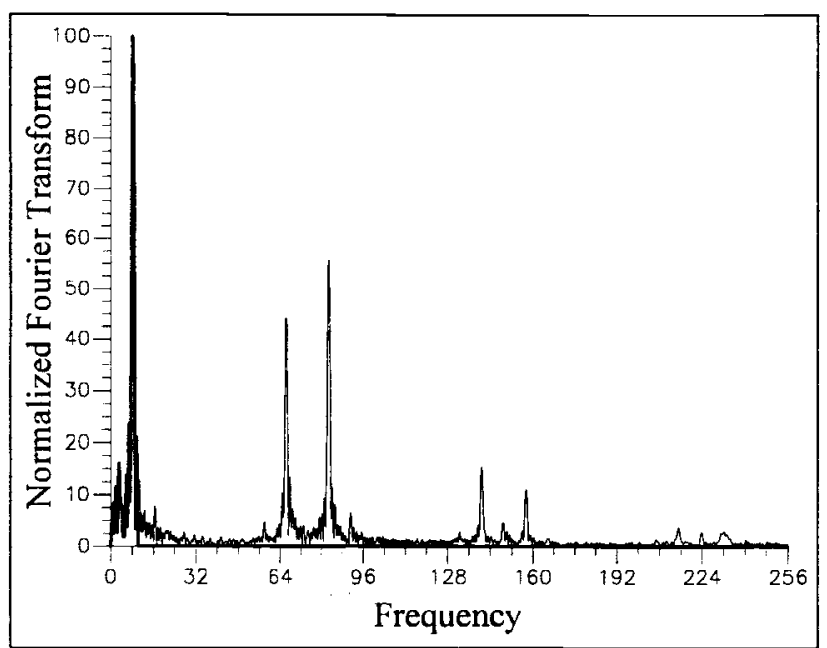

Figure 6. Fourier Transform of the Figure 4a function before (thinner line) and after a low pass filtering (thicker line)

value of several peaks has been considered. If the position of each peak $\left(d_{\text {peak }}\right)$ is expressed as an integer number $\left(n_{\text {peak }}\right)$ of periods $(P)$ plus a remainder $\left(R_{\text {peak }}\right)$ :

$$
d_{\text {peak }}=n_{\text {peak }} P+R_{\text {peak }}
$$

then, the right-left fringe displacement can be calculated as:

$$
\Delta=\frac{1}{M} \sum_{p e a k} R_{p e a k}
$$

where $M$ is the number of averaged peaks (if all of them are considered, $M=2 N / P$ ). The result for the signals presented in Figure 4 is $\Delta=29.5$ pixels.

\subsubsection{Period measurement}

The period of the fringes can be determined either from the left or right cosinusoidal distributions or from their correlation product. We have chosen the latter, which directly gives an average measure. The Fourier transform of the signal has to be computed and the frequency that gives a maximum value determined. By the relationship between $N$ and $P(P=N / f)$, an accuracy error $\epsilon_{f}$ in the frequency calculation is translated onto a period error, $\epsilon_{P}$ :

$$
\epsilon_{P}=\left|\frac{\partial P}{\partial f} \epsilon_{f}\right|=\frac{P^{2}}{N} \epsilon_{f}
$$

where all the errors are taken positive.

As a consequence of the Fast Fourier Transform routine employed for the calculations [28], the resolution error $\epsilon_{f}$ is the ratio between $N$ and the number of points of the signal to be transformed. As the correlation product has $2 N$ information terms, 


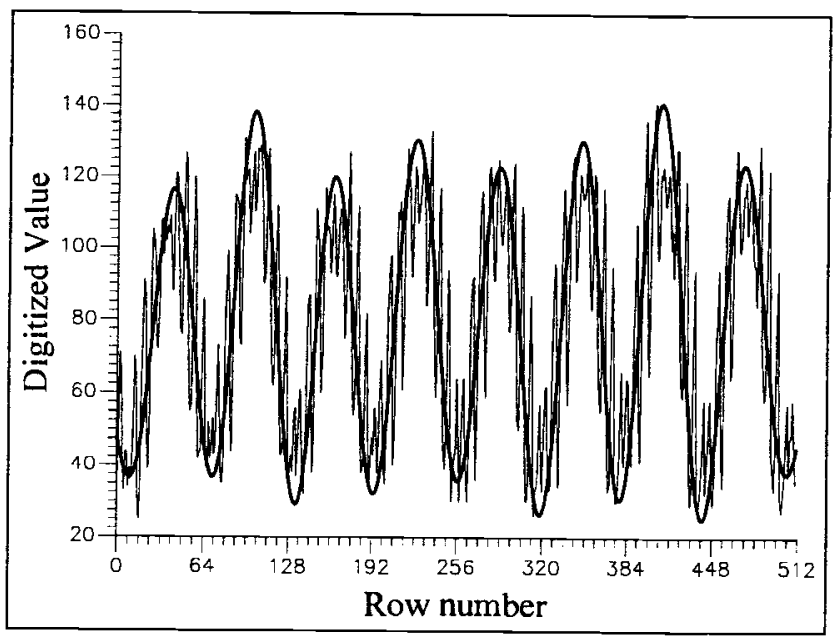

Figure 7. Cosinusoidal adjustement for the left distribution

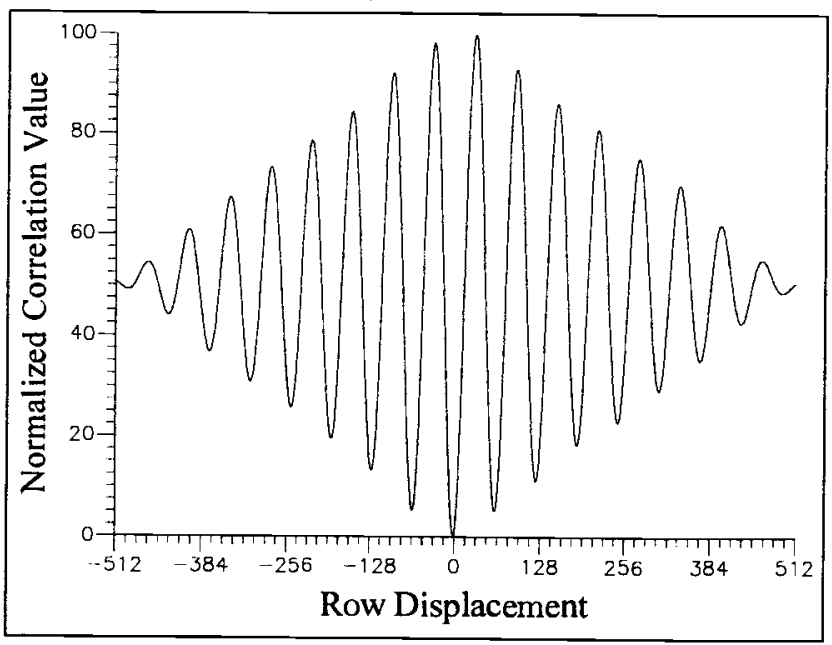

Figure 8. The correlation product between the cosinusoidal adjusted signals

$\epsilon_{f}=0.5$, which would give $\epsilon_{P}=16$ pixels for a period $P=64$. In order to reduce this error, it is possible to add non-data zeros to the Fourier transformation process so the total number of points increases, thus providing better resolution. We have considered that $\epsilon_{f}=1 / 64$ is an acceptable value for our calculations. In Figure 9, a Fourier transform comparison between the left and right fringe distributions and their correlation product is done. Note that while the left and right signals have a 0.25 frequency resolution ( $3 N$ zero points were added to the original signal), the correlation signal looks continuous, as the number of points is high. The period obtained from the Fourier Transform correlation function is $P=61.94$ pixels, so the final fringe shift is: $\phi=\frac{2 \pi \Delta}{P}=0.95 \pi$ radians $=171^{\circ}$ 


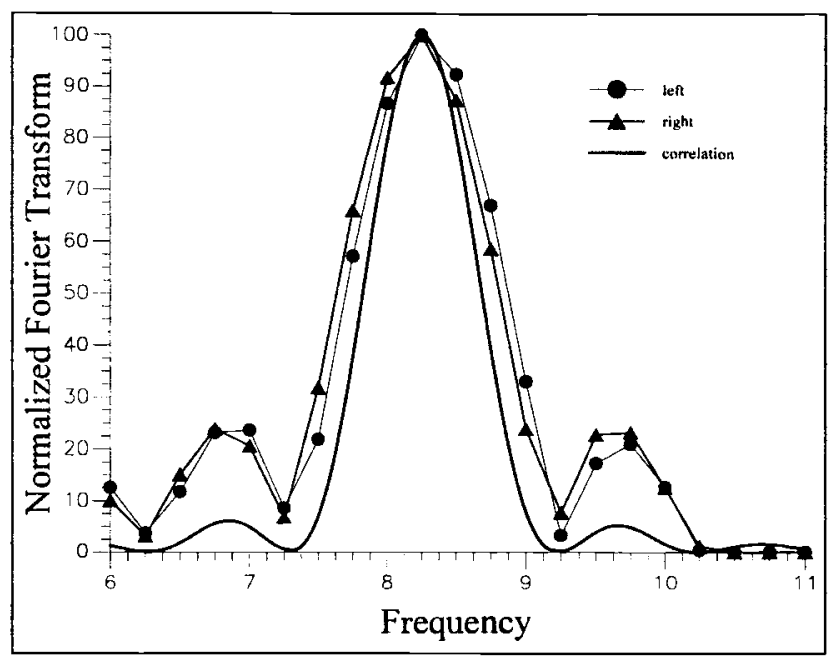

Figure 9. Fourier transform of the correlation product and the single signals

\subsection{Amplitude modulation method}

With the method described above, it is possible to determine the change in the light phase as it goes through the LCD. A related intensity light variation is always present. For a complete device calibration, it is then necessary to know its transmittance performance, usually called amplitude light modulation. The transmittance characteristics of the device are measured taking adavantage of the same setup as for the phase evaluation, in order to have identical experimental conditions (Figure 10). Note that only the right and upper path is used. An intensity detector, such as a luxmeter, which gives a linear output with respect to the intensity of the light, is placed first before the LCD and then after it. The whole working performance of the liquid crystal display involves both polarizers, so the second one has to be removed from its original position and placed between the LCD and the luxmeter. The transmittance of the device is the ratio between the intensity of the light before going through the panel and after it. The image displayed on the SLM is uniform, all the pixels having the same input grey level value. The measure is repeated for each of the possible input values, so the dependence of the transmittance with the grey level is determined. The amplitude modulation is simply given by the transmittance square root.

\subsection{Operating curves}

The amplitude and phase characteristics for a specific polarizers and potentiometers configuration can be jointly displayed on a complex plane curve, where each point stands for a single grey level, from $\mathrm{gl}=0$ to $\mathrm{gl}=255$. The modulus of a vector from the origin of coordinates to the considered point gives the amplitude modulation and the angle within the real-positive axis is the phase shift. The measure has been repeated for 32 evenly spaced grey-level values and the rest of them have been obtained by linear interpolation.

The choice of the operating curves for each LCD has to be taken into account to 


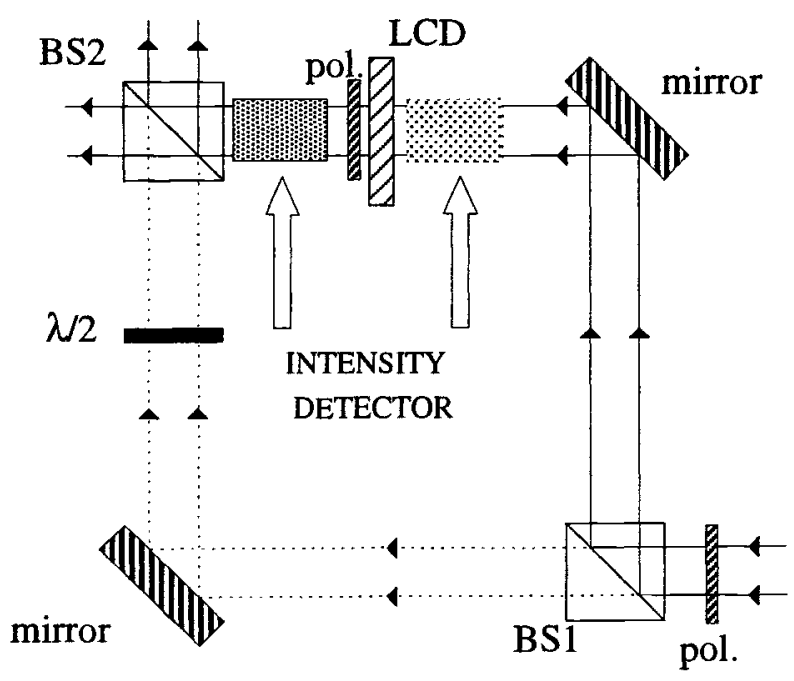

Figure 10. Experimental arrangement for the amplitude modulation measurement. The dashed path is not in use

optimize the results in the correlator $[29,30]$. The most common configurations used to display images on the LCDs are:

High Contrast (HC): In this configuration the LCD contrast ratio should be as high as possible, although there may be a significant phase modulation. The contrast is defined as $C=T_{\max } / T_{\min }$, where $T$ stands for the transmittance.

Amplitude Mostly (AM): In this case the LCD has to modulate light only in amplitude and the phase variation should be zero. In fact there is always a small phase modulation [10]. With this configuration the contrast ratio is usually much lower than with the $\mathrm{HC}$ one.

Phase Mostly $(\mathrm{PM})$ : Unlike the AM configuration, with this operating curve the light should be modulated only in phase but actually there is always a small variation in amplitude.

For correlation purposes, an $\mathrm{HC}$ configuration is required to display the input scene due to its good performance in contrast. The operating curve for the second LCD should be chosen according to the filter. In our case we want to display a phase-only filter (POF) [31], therefore we have to find a PM mode.

\subsection{Operating curves with linearly polarized light}

In Figures 11 and 12 we show two interesting LCD configuration results obtained with linearly polarized light both at the input and output sides of the panel. The orientations of the first polarizer and the analyzer (polarizer placed after the LCD) are indicated in degrees relative to laboratory vertical, positive if the polarizer turns clockwise in the direction of light propagation and negative if it turns counterclockwise. 
1999 Euro-American Workshop on Optoelectronic Information Processing / 83

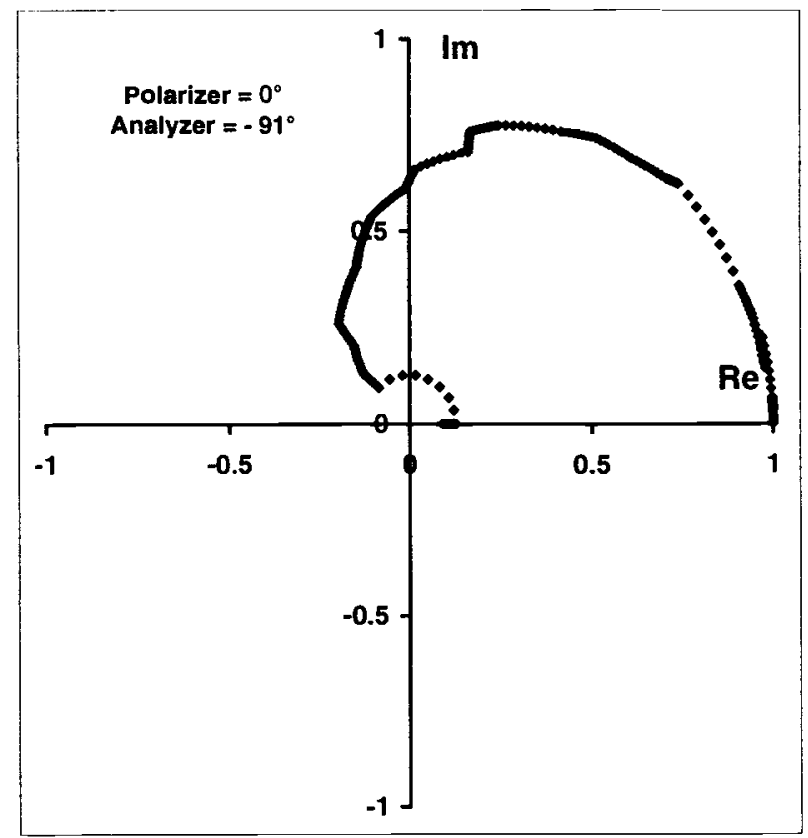

Figure 11. High contrast operating curve

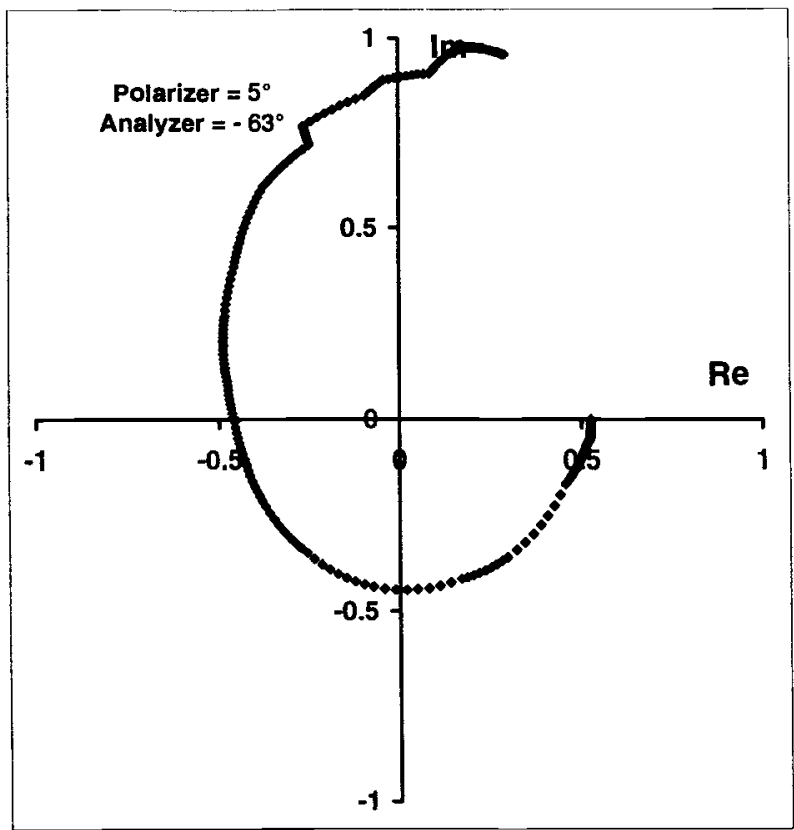

Figure 12. Phase-mostly operating curve

Proc. of SPIE Vol. 10296 1029605-13

Downloaded From: https://www.spiedigitallibrary.org/conference-proceedings-of-spie on 25 Apr 2023 Terms of Use: https://www.spiedigitallibrary.org/terms-of-use 
The first curve (Figure 11) corresponds to a HC mode, in which the device transmittance (amplitude square) increases with the grey level, reaching a normalized maximum value of 1 for $\mathrm{gl}=255$. The contrast achieved with this configuration is about 125:1.

The second one (Figure 12) is the best PM operating curve we have obtained. It is not advisable to use this configuration to display a phase-only filter for several reasons:

1. The phase modulation only reaches $1.6 \pi$ radians

2. The amplitude modulation is not constant. The minimum value is about $43 \%$ of the maximum.

3 . If we use only the $0-\pi$ range, the transmittance is too low to get good correlation results and we lose bandwidth (104 grey levels).

As pointed out in certain papers $[29,32,33]$, if we use elliptically polarized light it will be possible to find a configuration with only phase modulation.

\subsection{Operating curves with elliptically polarized light}

If a linear polarizer plus a $\lambda / 4$ plate are used instead of a linear polarizer, elliptically polarized light can be generated. In consequence, now the LCD is placed between a couple of sytems made of a linear polarizer plus a quarter-wave plate, as shown in Figure 13.

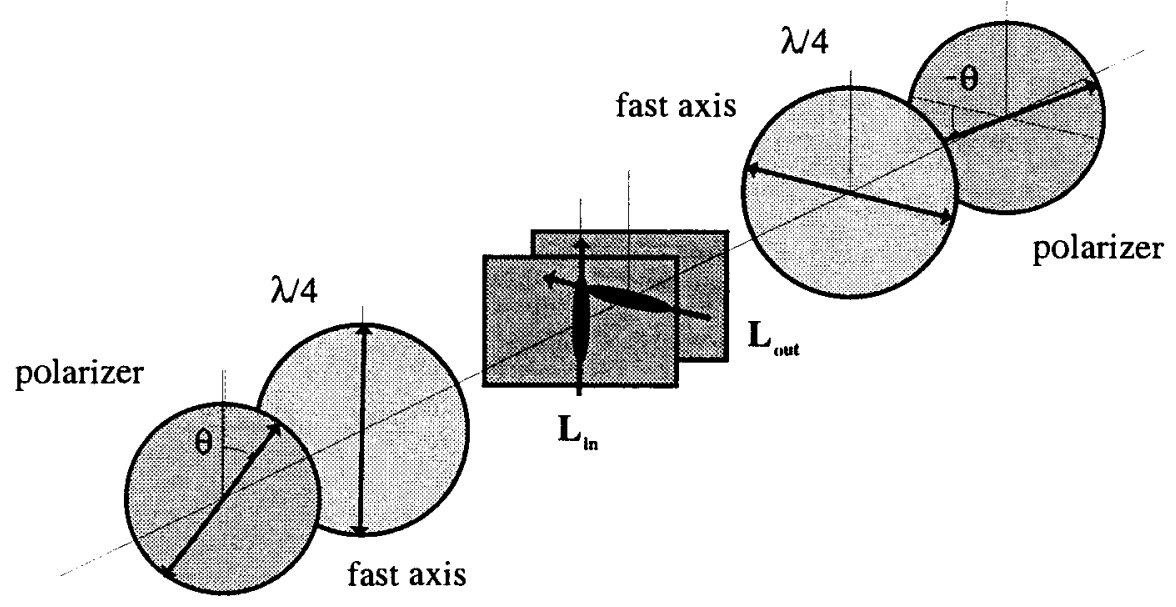

Figure 13. Experimental setup for elliptically polarized light

In order to determine the right orientation of the elements to achieve only phase modulation we have to find the parameters of the LCD, such as the molecular directors $\left(\mathbf{L}_{\mathbf{i n}}\right.$ and $\left.\mathbf{L}_{\text {out }}\right)$ and the twist angle $(\alpha)$. Following the procedure explained in [34] and taking into account the corrections suggested in [35], we have obtained for the red panel $\mathbf{L}_{\mathrm{in}}=1^{\circ}, \mathbf{L}_{\text {out }}=-76^{\circ}$ and $\alpha=-77^{\circ}$. The orientations are 
indicated in degrees relative to laboratory vertical, taking the clockwise direction as positive.

First, the fast axis of the quarter-wave plates must be orientated parallel to the corresponding molecular directors at each side of the panel. Then, we have to rotate the linear polarizers to get the best phase-mostly configuration. Following [33], the first one has to be alligned at an angle $\theta$ relatively to the molecular director at the input side of the LCD, while the second one has to be orientated at $-\theta$ relatively to the output molecular director. The value obtained for our LCD is $\theta=35^{\circ}$, giving the operating curve shown in Figure 14.

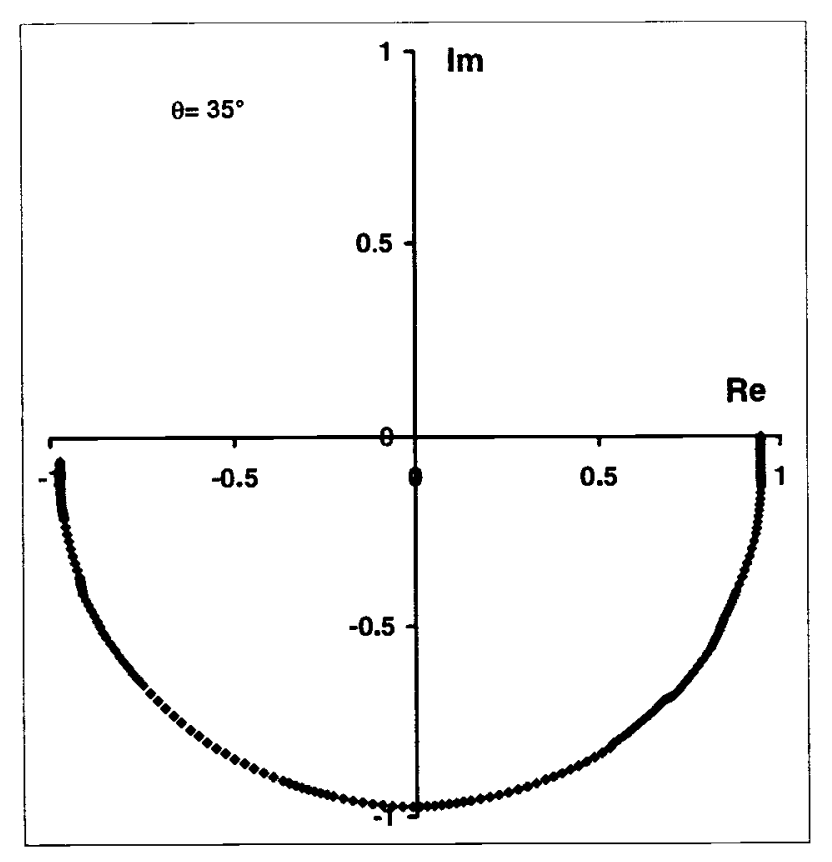

Figure 14. 0- $\pi$ modulation operating curve of the LCD

This configuration allows the use of the 256 grey levels available with a phase-only modulation and a constant transmittance. Although this operational mode only reaches $\pi$ radians, we show in the next section a way to overcome this drawback for correlation purposes.

\section{IMPLEMENTATION OF PHASE-ONLY FILTERS USING $[0-\pi]$ MODULATION}

We have noticed that our devices can operate in a phase-only modulation configuration in the range $[0-\pi]$ with constant amplitude and using all the dynamic range of the image. In such conditions we will show that it is possible to achieve the same correlations than the ones obtained with an ordinary POF. Let $f(x, y)$ be the reference to detect, and let $F(u, v)=|F(u, v)| \exp (-i \phi(u, v))$ be its Fourier 
86 / Critical Reviews Vol. CR74

transform, the POF is defined as:

$$
F_{P O F}(u, v)=\frac{F^{*}(u, v)}{|F(u, v)|}=\exp (-i \phi(u, v))
$$

We introduce the $\pi$ phase-only filter $(\pi-\mathrm{POF})$ as:

$$
\begin{aligned}
F_{\pi-P O F}(u, v)=\exp (-i|\phi(u, v)|) & =\cos (|\phi(u, v)|)-i \sin (|\phi(u, v)|)= \\
& =\cos (\phi(u, v))-i|\sin (\phi(u, v))|
\end{aligned}
$$

The function $|\sin (x)|$, can be written as a Fourier series as follows:

$$
|\sin (x)|=\frac{2}{\pi}-\frac{4}{\pi}\left[\frac{1}{3} \cos (2 x)+\frac{1}{15} \cos (4 x)+\frac{1}{35} \cos (6 x)+\ldots\right]
$$

therefore the phase codified in the filter can be written as:

$$
\exp (-i|\phi(u, v)|)=\cos (\phi(u, v))-\frac{2 i}{\pi}+\frac{4 i}{3 \pi} \cos (2 \phi(u, v))+\frac{4 i}{15 \pi} \cos (4 \phi(u, v))+\ldots
$$

In order to avoid the difraction of all the terms superimposed on the zero order, a linear phase shift is introduced in the expression of the filter:

$$
\begin{array}{r}
\exp (-i|\phi(u, v)+2 \pi a u|)=\cos (\phi(u, v)+2 \pi a u)-\frac{2 i}{\pi}+ \\
+\frac{4 i}{3 \pi} \cos (2 \phi(u, v)+2 \pi(2 a) u)+\frac{4 i}{15 \pi} \cos (4 \phi(u, v)+2 \pi(4 a) u)+\ldots
\end{array}
$$

Analyzing the aforementioned equation, we can get the information about the filter reconstruction. Using the $\pi-\mathrm{POF}$, we have an on-axis constant term and simetrically arranged from it, at a distance $x= \pm a$, there are the phase-only reconstructions of the reference. These images have their phases conjugated. There are also higher order harmonics with a decreasing energy at the points $x= \pm 2 a, \pm 4 a, \pm 6 a \ldots$ Therefore, it is expected to find the correlation and convolution between the scene and the ordinary POF $(\exp (i \phi(u, v)))$ situated simetrically from the on-axis reconstruction of the scene.
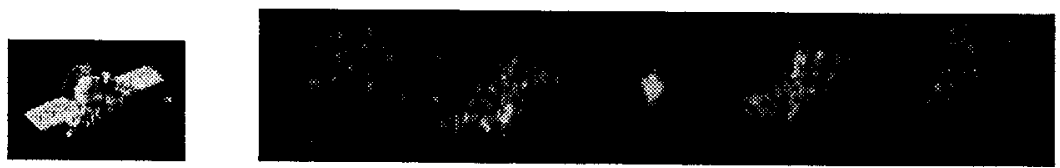

Figure 15. Reference and reconstruction of the $\pi$-POF adapted to it

To demonstrate experimentally the latter analysis, in Figure 15 we show the reference and the experimental reconstruction of the $\pi$-POF adapted to it. The centered constant term can be clearly seen and the two phase terms, that will produce the correlation and convolution between the target and the scene, appear equally separated from the center. Second order harmonics are observable at a double distance. 


\section{OPTICAL RESULTS}

In this section we demonstrate the use of the optical correlator we have built with the two LCDs at the input and the Fourier planes. On the righthand side of Figures 16,17 and 18, we show the relevant area of the three satellite scene used in the experiments.
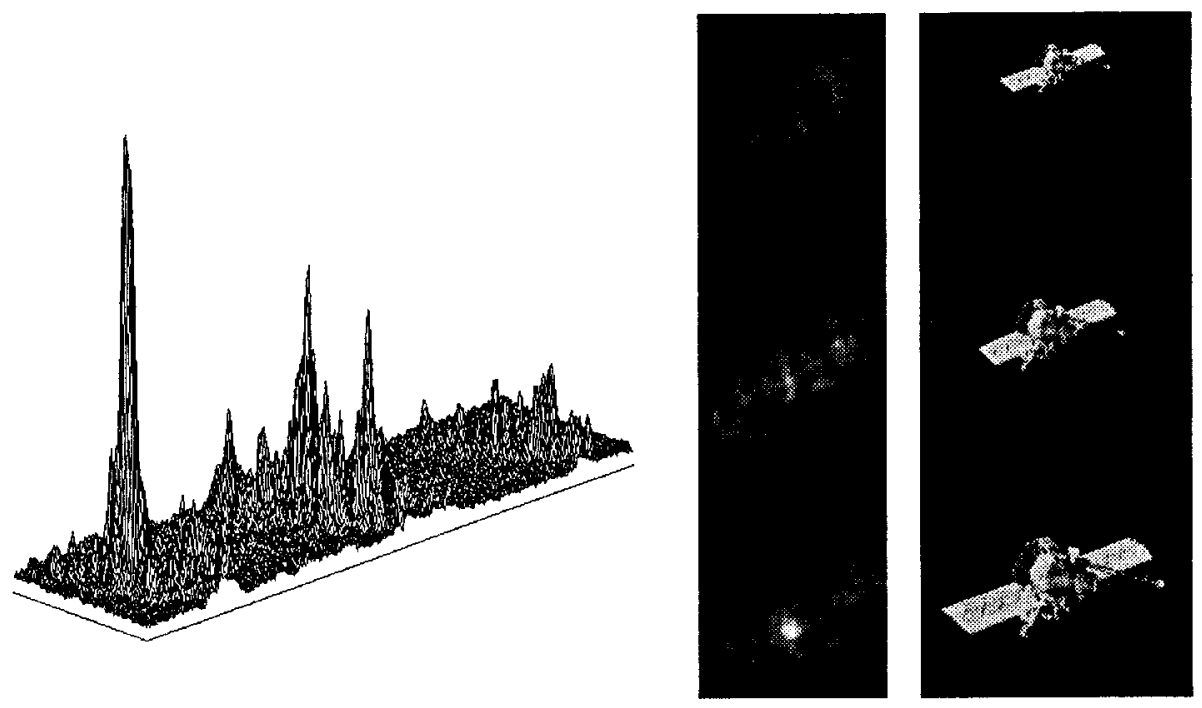

Figure 16. Correlation between the scene and the largest satellite
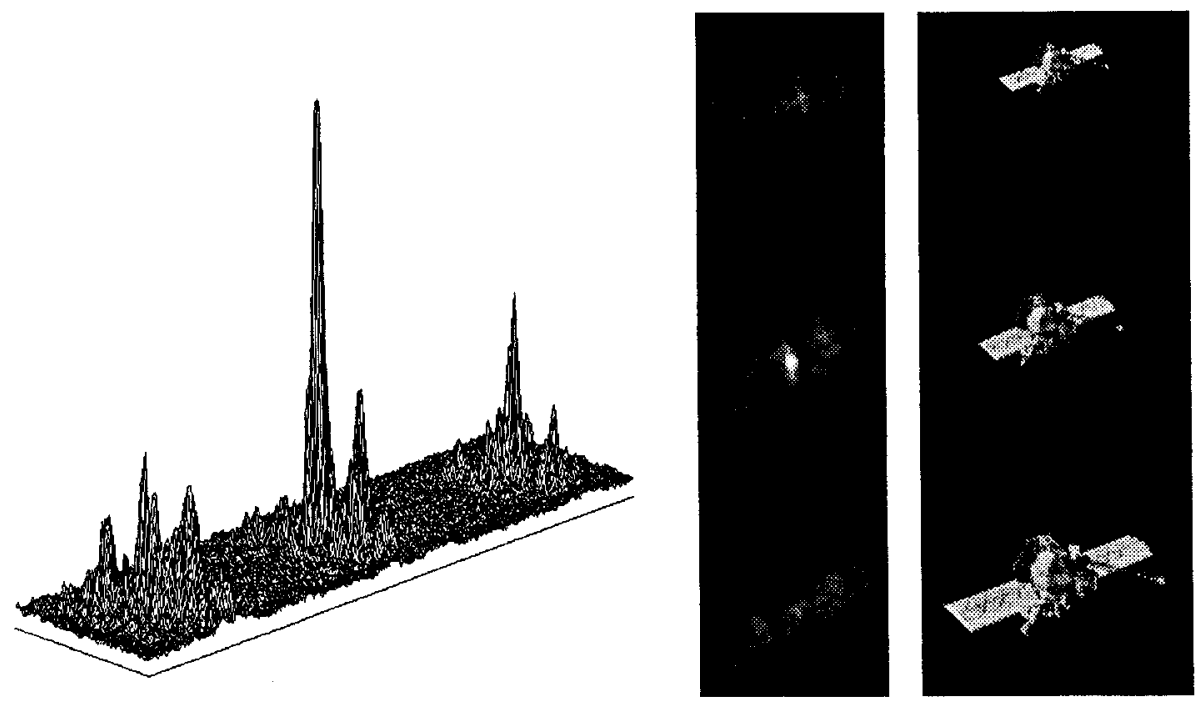

Figure 17. Correlation between the scene and the medium satellite

Proc. of SPIE Vol. 10296 1029605-17 

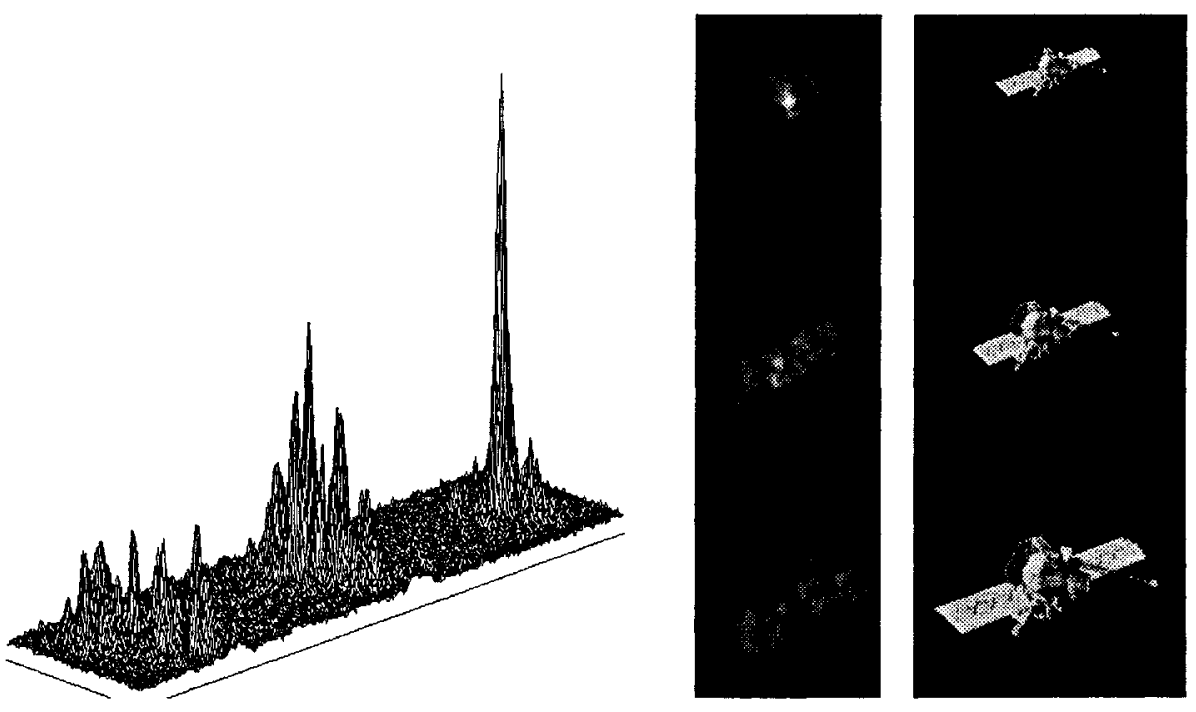

Figure 18. Correlation between the scene and the smallest satellite

This image, adapted to the VGA resolution by adding zero values, is displayed as the scene on the first LCD working with a high contrast $(\mathrm{HC})$ operating curve. The second panel is in the $[0-\pi]$ phase-only modulation configuration in order to display the filter. The $\pi$-POF of the target has been computed taking into account the phase introduced in the scene by the HC configuration of the first LCD. Figures 16, 17 and 18 show the optical correlation between the scene and the three different satellites used as the reference to be detected, respectively. The experimental results show a good performance of the procedure developed.

\section{CONCLUSIONS}

We have implemented a converging VanderLugt correlator that makes use of two VGA LCDs to display the images. These panels have been detached from their original mounts and subsequently characterized following an interferometric procedure which includes the amplitude and phase response measurement. The characterization method has been use with the aim of achieving suitable operating curves which depend on both the electronical and optical work conditions. We have presented a high contrast operating curve and a selection between two phase-mostly configurations, by using linearly and elliptically polarized light, respectively. We have proposed a modification of the ordinary POF, by taking the absolute value of the phase in order to be displayed on panels with partial phase-only modulation. Experimental results have shown the good performance of the method.

\section{ACKNOWLEDGMENTS}

This work is supported by CICYT (Comisión Interministerial de Ciencia y Tecnología) under the project TAP97-0454. 


\section{REFERENCES}

1. U. Efron, "Spatial light modulators and applications for optical information processing", Proc. SPIE 960, pp. 180-203, 1988.

2. D. A. Gregory, "Real-time pattern recognition using a modified liquid crystal television in a coherent optical correlator", Appl. Opt. 25, pp. 467-469, 1986.

3. N. Clark, C. M. Crandall, and M. K. Giles, "Using liquid crystal TVs in Vander Lugt optical correlators", Proc. SPIE 1564, pp. 439-451, 1991.

4. A. Stoianov, C. Soutar, and A. Graham, "High-speed fingerprint verification using an optical correlator", Proc. SPIE 3386, pp. 242-252, 1998.

5. J. C. Kirsch, D. A. Gregory, M. W. Thie, and B. K. Jones, "Modulation characteristics of the Epson liquid crystal television", Opt. Eng. 31, pp. 963-970, 1992.

6. J. N. Duffey, B. K. Jones, J. A. Loudin, and J. Booth, "Optical characterization of the InFocus TVT-600 LCTV using custom device electronics", Proc. SPIE 2490, pp. 432-440, 1995.

7. J. P. Karins, S. A. Mills, N. J. Szegedi, J. R. Ryan, L. G. Kelly, D. H. Goldstein, E. P. Augustus, and R. J. Wrangler, "Miniature ruggedized optical correlator for flight testing", Proc. SPIE 2237, pp. 48-53, 1994.

8. B. K. Jones and J. C. Kirsch, "Optical correlator comparison of near and far field ISAR imagery", Proc. SPIE 2490, pp. 312-317, 1995.

9. H. Liu, J. Davis, and R. Lilly, "Optical-data-processing properties of a liquid crystal television spatial light modulator", Opt. Lett. 10, pp. 635-637, 1985.

10. K. Lu and B. E. A. Saleh, "Theory and design of the liquid crystal TV as an optical spatial phase modulator", Opt. Eng. 29, pp. 240-246, 1990.

11. B. Javidi and J. Horner, "Optical pattern recognition for validation and security verification", Opt. Eng. 33, pp. 1752-1756, 1994.

12. V. Laude, S. Mazé, P. Chavel, and P. Réfrégier, "Amplitude and phase coding measurements of a liquid crystal television", Opt. Commun. 103, pp. 33-38, 1993.

13. C. Soutar, S. E. Monroe, and J. Knopp, "Measurement of the complex transmittance of the Epson liquid crystal television", Opt. Eng. 33, pp. 1061-1068, 1994.

14. Z. Zhang, G. Lu, and F. Yu, "Simple method for measuring phase modulation in liquid crystal televisions", Opt. Eng. 33, pp. 3018-3022, 1994.

15. J. L. McClain, P. S. Erbach, D. A. Gregory, and F. T. S. Yu, "Spatial light modulators phase depth determination from optical diffraction information", Opt. Eng. 35, pp. 951-954, 1996.

16. A. B. VanderLugt, "Signal detection by complex filtering", IEEE Transactions on Information Theory IT-10, pp. 139-145, 1964.

17. D. Joyeux and S. Lowenthal, "Optical Fourier transform: what is the optimal setup ?", Appl. Opt. 21, pp. 4368-4372, 1982.

18. D. Flannery, A. M. Biernacki, J. S. Loomis, and S. L. Cartwright, "Real-time coherent correlator using binary magnetooptic spatial light modulators at input and Fourier planes", Appl. Opt. 25, p. 466, 1986.

19. B. D. Bock, T. A. Crow, and M. K. Giles, "Design considerations for miniature optical correlation systems that use pixelated input and filter transducers", Proc. SPIE 1347, pp. 297-309, 1990. 
90 / Critical Reviews Vol. CR74

20. I. Juvells, S. Vallmitjana, and S. Bosch, "Analysis of a scale-tunable telephoto lens diffractometer and its use in optical correlation", J. Modern Optics 39, pp. 1107-1115, 1992.

21. M. Montes-Usategui, S. E. Monroe, and R. D. Juday, "Automated selfalignement procedure for optical correlators", Opt. Eng. 36, pp. 1782-1791, 1997.

22. A. B. VanderLugt, "Operational notation for the analyisis of optical dataprocessing systems", Proc. IEEE 54, pp. 1055-1062, 1966.

23. Y. Sheng and G. Paul-Hus, "Optical on-axis imperfect phase-only correlator using liquid-crystal television", Appl. Opt. 32, pp. 5782-5785, 1993.

24. L. Gonçalves, D. Roberge, and Y. Sheng, "Programmable optical phase-mostly holograms with coupled-mode modulation liquid-crystal television", Appl. Opt. 34, pp. 1944-1950, 1995.

25. E. Martín-Badosa, A. Carnicer, I. Juvells, and S. Vallmitjana, "Liquid crystal devices complex modulation characterization by interferometric data correlation", Meas. Sci. Technol 8, pp. 764-772, 1997.

26. M. Born and E. Wolf, Principles of optics, Pergamon Press, Oxford, 1980.

27. M. Takeda, H. Ida, and S. Kobayashi, "Fourier-transform method of fringepattern analysis for computer-based topography and interferometry", J. Opt. Soc. Am. 72, pp. 156-160, 1982.

28. E. O. Brigham, The Fast Fourier Transform and its applications, PrenticeHall, New Jersey, 1988.

29. C. Soutar and S. E. Monroe, "Selection of operating curves of twisted-nematic liquid crystal televisions", Proc. SPIE 2240, pp. 280-291, 1994.

30. A. Skorucak and C. Soutar, "Selection criteria for spatial light modulator operating curves", Proc. SPIE 2752, pp. 120-131, 1996.

31. J. L. Horner and P. D. Gianino, "Phase-only matched filtering", Appl. Opt. 23, pp. 812-816, 1984.

32. J. L. Pezzaniti and R. A. Chipman, "Phase-only modulation of a twisted nematic liquid-crystal tv by use of the eigenpolarization states", Opt. Lett. 18, pp. 1567-1569, 1993.

33. J. A. Davis, I. Moreno, and P. Tsai, "Polarization eigenstates for twistednematic liquid crystals displays", Appl. Opt. 37, pp. 937-945, 1998.

34. M. Yamauchi and T. Eiju, "Optimiztion of twisted nematic liquid cristal panels for spatial light modulation", Opt. Commun. 115, pp. 19-25, 1995.

35. J. A. Coy, M. Zaldarriaga, D. F. Grosz, and O. E. Martínez, "Characterization of a liquid crystal television as a programmable spatial light modulator", Opt. Eng. 35, pp. 15-19, 1996. 